\title{
Supergravity with mimetic dark matter
}

\author{
Ali H. Chamseddine \\ Physics Department, American University of Beirut, Lebanon
}

\begin{abstract}
We formulate a supersymmetric version of gravity with mimetic dark matter. The coupling of a constrained chiral multiplet to $\mathrm{N}=1$ supergravity is made locally supersymmetric using the rules of tensor calculus. The chiral multiplet is constrained with a Lagrange multiplier multiplet that could be either a chiral multiplet or a linear multiplet. We obtain the fully supersymmetric Lagrangians in both cases. It is then shown that the system consisting of the supergravity multiplet, the chiral multiplet and the Lagrange multiplier multiplet can break supersymmetry spontaneously leading to a model of a graviton, massive gravitino and two scalar fields representing mimetic dark matter. The combination of the chiral multiplet and Lagrange multiplier multiplet can act as the hidden sector breaking local $\mathrm{N}=1$ supersymmetry.
\end{abstract}




\section{Introduction}

The role of diffeomorphism invariance in General Relativity is to promote redundancy in presentation of the dynamical degrees of freedom in exchange of gaining simplicity and elegancy in the formulation. A metric $g_{\mu \nu}$ with ten components is used to represent two dynamical degrees of freedom for the graviton. It is then natural to seek a modified theory of gravity without increasing the degrees of freedom of the system while preserving diffeomorphism invariance. Some years ago such a modified theory of gravity was proposed by Mukhanov and myself [1], based on the idea of employing a dynamical metric $g_{\mu \nu}$ depending on an auxiliary metric $\widetilde{g}_{\mu \nu}$ through the relation

$$
g_{\mu \nu}=\widetilde{g}_{\mu \nu} \widetilde{g}^{\kappa \lambda} \partial_{\kappa} \phi \partial_{\lambda} \phi,
$$

where $\phi$ is an additional scalar field. This definition exhibits scale invariance under the transformation

$$
\widetilde{g}_{\mu \nu} \rightarrow \widetilde{g}_{\mu \nu} e^{\omega(x)},
$$

suggesting a relation between the field $\phi$ and the scale factor of $g_{\mu \nu}$ as can be seen from the property

$$
g^{\mu \nu} \partial_{\mu} \phi \partial_{\nu} \phi=1
$$

Equivalently, one can show that the general solution of the constraint equation (3) is given by (11). It is easier to impose the constraint (3) through a Lagrange multiplier than to work with an auxiliary metric $\widetilde{g}_{\mu \nu}[2]$. It proved that the simple observation of formulating a theory of gravity subject to the constraint (3) has many important consequences. First, it was shown that the system $\left(g_{\mu \nu}, \phi\right)$ with eleven fields constrained by equation (3) is equivalent to GR with an additional half of a degree of freedom for the longitudinal mode that contributes the equivalent of pressureless dust to the energy-momentum tensor [1]. Next, it was seen that by adding a potential function $V(\phi)$ it is possible to construct various physical cosmological models [3]. In particular, the field $\phi$ could serve as the inflaton field. In the synchronous gauge a solution of the constraint equation (3) is

$$
\phi=t+c,
$$

and thus the field $\phi$ represents the time coordinate. In general for an arbitrary metric $g_{\mu \nu}$ the constraint equation (3) defines the field $\phi$ as that of synchronous time. For the solution (4) we have $\square \phi=\kappa$ where $\kappa$ is the extrinsic curvature, it was then shown that by adding to the Einstein-Hilbert 
action terms of the form $f(\square \phi)$ for some suitable functions $f$, it is possible to resolve cosmological singularities [4]. The function $f(\square \phi)$ contributes to the energy momentum tensor and acts as a source of "mimetic" dark matter providing a geometrical explanation of dark matter without the need of introducing new forms of interaction. Exact solutions of the system of equations with a smooth metric at $t=0$ were found for Friedmann, Kasner universes as well as for black holes [5], 6], [7].

On the other hand, supersymmetry, since the 1970's, has played a prominent role in the attempts to promote gravity to a finite quantum theory within the settings of supergravity and superstring theory in various dimensions ranging from four to eleven. Supersymmetry, however, is not observed in nature and must be spontaneously broken. In most realistic models this is done in the context of four dimensional $N=1$ supergravity with the gravitino absorbing a Goldistino fermion [8]. The purpose of this article is to construct a supersymmetric form of gravity with mimetic dark matter. This will be done using the methods of $N=1$ supersymmetric tensor calculus [9], [8], [10, [11, [12], [13]. It turns out that there are few number of possibilities depending on the type of supermultiplet used for the Lagrange multiplier or the one including the field $\phi$. In this paper we will explore two possibilities. In the first, a chiral multiplet is used as a Lagrange multiplier multiplet to impose a constraint on the metric coupled to a chiral multiplet. The constraint on the scalar field $z$ is of the form $z \square z+\bar{z} \square \bar{z}$ instead of $g^{\mu \nu} \partial_{\mu} z \partial_{\nu} \bar{z}$. This is done in section 2 . In section 3 a linear multiplet is used instead as the Lagrange multiplier multiplet, and results in a constraint on the kinetic term $g^{\mu \nu} \partial_{\mu} z \partial_{\nu} \bar{z}$ in line with the non-supersymmetric case. Section 4 is the conclusion and section 5 gives the full supersymmetric Lagrangians in component form for the two cases considered.

\section{Supergravity with mimetic dark matter}

To find the locally superysmmetric form of supergravity with mimetic matter we have to first specify the nature of the supermultiplets used. In what follows we will use the notation and conventions of [11], [13. The constraint term (31) is the kinetic part of a real scalar field can be embedded as part of a left-handed chiral multiplet $\Sigma$ denoted by

$$
\Sigma=(z, \chi, h),
$$

with the multiplet of opposite chirality given by

$$
\Sigma^{\dagger}=\left(\bar{z}, \chi^{c}, \bar{h}\right),
$$


where $\bar{z}, \bar{h}$ are the complex conjugates of $z, h$, and $\chi^{c}=C^{-1} \bar{\chi}$ is the spinor conjugate to $\chi$. The symmetric product $\frac{1}{2} \Sigma^{\dagger} \Sigma$ is a real vector multiplet of the form

$$
V=\left(C, \zeta, v, V_{\mu}, \lambda, D\right)
$$

where $C, D$ are real scalar fields and $v$ is a complex scalar field, $\zeta, \lambda$ are Majorana spinors and $V_{\mu}$ is a real vector. To deal with the most general coupling we consider a function $\phi\left(\Sigma, \Sigma^{\dagger}\right)$ of the symmetric products of $\Sigma^{\dagger}$ and $\Sigma$ whose lowest component is

$$
\phi(z, \bar{z})=\sum_{m, n} a_{m, n} z^{m} \bar{z}^{n} .
$$

The components of the real vector multiplet are given by

$$
\begin{aligned}
C & =\frac{1}{2} \phi, \quad \zeta=i\left(\phi_{, z} \chi-\phi_{, \bar{z}} \chi^{c}\right) \\
v & =-\phi_{, z} h+\phi_{, z z} \overline{\chi^{c}} \chi, \quad V_{\mu}=\frac{i}{2}\left(\phi_{, z} \widehat{D}_{\mu} z-\phi_{, \bar{z}} \widehat{D}_{\mu} \bar{z}-2 \phi_{, z \bar{z}} \bar{\chi} \gamma_{\mu} \chi\right) \\
\lambda & =-i \phi_{, z \bar{z}}\left(\left(\bar{h} \chi-h \chi^{c}\right)+\widehat{D} \bar{z} \chi-\widehat{D} z \chi^{c}\right)-i \phi_{, z z \bar{z}}\left(\overline{\chi^{c}} \chi\right) \chi^{c}+i \phi_{, z \overline{z z}}\left(\bar{\chi} \chi^{c}\right) \chi \\
D & =\phi_{, z \bar{z}}\left(|h|^{2}-\widehat{D}_{\mu} \bar{z} \widehat{D}^{\mu} z-\bar{\chi} \overleftrightarrow{D^{\prime}} \chi\right)-\phi_{, z z \bar{z}}\left(\bar{h} \overline{\chi^{c}} \chi+\bar{\chi} \gamma^{\mu} \chi \widehat{D}_{\mu} z\right) \\
& -\phi_{, z \overline{z z}}\left(h \bar{\chi} \chi^{c}+\overline{\chi^{c}} \gamma^{\mu} \chi^{c} \widehat{D}_{\mu} \bar{z}\right)+\phi_{, z z \overline{z z}}\left(\overline{\chi^{c}} \chi\right)\left(\bar{\chi} \chi^{c}\right) .
\end{aligned}
$$

We recognize that the $D$-term of the above vector multiplet contains the kinetic term of the complex scalar field $z$ and for this to be constrained by a Lagrange multiplier it must couple to a real vector multiplet. The most economical choice is to use a left-handed chiral multiplet

$$
S=(A+i B, \psi, F+i G)
$$

that can be embedded in a real irreducible vector multiplet of the form [14]

$$
\widehat{D} S=\left(B, \psi, l, \widehat{D}_{\mu} A, 0,0\right)
$$

where $l=F+i G$. The rule of multiplying two vector multiplets $V=V_{1} \cdot V_{2}$ where $V_{1}=\left(C_{1}, \zeta_{1}, v_{1}, V_{\mu 1}, \lambda_{1}, D_{1}\right)$ and $V_{2}=\left(C_{2}, \zeta_{2}, v_{2}, V_{\mu 2}, \lambda_{2}, D_{2}\right)$ is given 
by $[9]$, 13

$$
\begin{aligned}
C & =C_{1} C_{2}, \quad \zeta=C_{1} \zeta_{2}+C_{2} \zeta_{1} \\
v & =C_{1} v_{2}+C_{2} v_{1}-\frac{1}{2} \bar{\zeta}_{1 R} \zeta_{2 L}-\frac{1}{2} \bar{\zeta}_{2 R} \zeta_{1 L} \\
V_{\mu} & =C_{1} V_{\mu 2}+C_{2} V_{\mu 1}-\frac{i}{2} \bar{\zeta}_{1} \gamma_{\mu} \gamma_{5} \zeta_{2} \\
\lambda & =C_{1} \lambda_{2}-\frac{1}{2} \widehat{D} C_{1} \zeta_{2}+\frac{1}{2} \widehat{\bar{v}}_{1} \zeta_{2}+\frac{i}{2} \gamma_{5} \gamma^{\mu} \zeta_{2} V_{\mu 1}+1 \leftrightarrow 2 \\
D & =C_{1} D_{2}-\frac{1}{2} \widehat{D}_{\mu} C_{1} \widehat{D}^{\mu} C_{2}-\frac{1}{2} V_{\mu 1} V_{2}^{\mu}+\frac{1}{2} \bar{v}_{1} v_{2}-\bar{\zeta}_{1} \lambda_{2}-\frac{1}{2} \bar{\zeta}_{2} \widehat{D D} \zeta_{1}+1 \leftrightarrow 2
\end{aligned}
$$

where the various quantities are defined in Reference [13. The coupling of minimal $N=1$ supergravity to a vector multiplet is given by the Lagrangian [9], [8]

$$
\begin{aligned}
e^{-1} L_{D} & =D-\frac{i}{2} \bar{\psi}_{\mu} \gamma_{5} \gamma^{\mu} \lambda-\frac{1}{3}(\bar{u} v+u \bar{v}) \\
& +\frac{2}{3} V_{\mu}\left(A^{\mu}+\frac{3 i}{8} e^{-1} \epsilon^{\mu \rho \sigma \tau} \bar{\psi}_{\rho} \gamma_{\tau} \psi_{\sigma}\right) \\
& +\frac{i}{3} e^{-1} \bar{\zeta} \gamma_{5} \gamma_{\mu} R^{\mu}+\frac{i}{8} e^{-1} \epsilon^{\mu \nu \rho \sigma} \bar{\psi}_{\mu} \gamma_{\nu} \psi_{\rho} \bar{\zeta} \psi_{\sigma} \\
& -\frac{2}{3} e^{-1} C L_{S G},
\end{aligned}
$$

where

$$
e^{-1} L_{S G}=-\frac{1}{2} R(e, \omega)-\frac{1}{3}|u|^{2}+\frac{1}{3} A_{\mu} A^{\mu}-\frac{1}{2} e^{-1} \bar{\psi}_{\mu} R^{\mu} .
$$

The fields $u=S-i P$ and $A_{\mu}$ are auxiliary fields and

$$
R^{\mu}=\epsilon^{\mu \nu \rho \sigma} \gamma_{5} \gamma_{\nu} D_{\rho}(\omega) \psi_{\sigma}
$$


Examining the components of the product vector multiplet $\frac{1}{2}\left(\widehat{D} S \cdot \phi\left(\Sigma, \Sigma^{\dagger}\right)\right)$ we obtain [13]

$$
\begin{aligned}
& C=\frac{1}{2} B \phi, \quad \xi=i B\left(\phi_{, z} \chi-\phi, \bar{z} \chi^{c}\right)+\frac{1}{2} \phi \psi, \\
& v=-B\left(h \phi_{, z}-\phi_{, z z} \overline{\chi^{c}} \chi\right)+\frac{1}{2} \phi l-\frac{i}{2} \bar{\psi}_{R}\left(\phi_{, z} \chi_{L}-\phi_{, \bar{z}} \chi_{L}^{c}\right)+\frac{i}{2}\left(\phi_{, \bar{z}} \bar{\chi}_{R}-\phi_{, z} \bar{\chi}_{R}{ }_{R}\right) \psi_{L}, \\
& V_{\mu}=\frac{i}{2} B\left(\phi_{, z} \widehat{D}_{\mu} z-\phi_{, \bar{z}} \widehat{D}_{\mu} \bar{z}-2 \phi_{, z \bar{z}} \bar{\chi} \gamma_{\mu} \chi\right)+\frac{1}{2} \phi \widehat{D}_{\mu} A+\frac{1}{2} \bar{\psi} \gamma_{\mu} \gamma_{5}\left(\phi_{, z} \chi-\phi_{, \bar{z}} \chi^{c}\right), \\
& \lambda=-i B \phi_{, z z}\left(\left(\bar{h} \chi-h \chi^{c}\right)+\widehat{D D} \bar{z} \chi-\widehat{D} z \chi^{c}\right) \\
& -i B\left(\phi_{, z z \bar{z}}\left(\overline{\chi^{c}} \chi\right) \chi^{c}-\phi_{, z \overline{z z}}\left(\bar{\chi} \chi^{c}\right) \chi\right)-\frac{i}{2} \widehat{\not D} B\left(\phi_{, z} \chi-\phi_{, \bar{z}} \chi^{c}\right) \\
& -\frac{1}{4} \widehat{D} \phi \psi-\frac{1}{2} \gamma_{5} \gamma^{\mu}\left(\phi_{, z} \chi-\phi, \bar{z} \chi^{c}\right) \widehat{D}_{\mu} A-\frac{1}{2}\left(\phi_{, \bar{z}} \bar{h} \widehat{-\phi_{, z z}} \overline{\chi^{c}} \chi\right) \psi \\
& -\frac{1}{4} \gamma_{5} \gamma^{\mu} \psi\left(\phi_{, z} \widehat{D}_{\mu} z-\phi_{, \bar{z}} \widehat{D}_{\mu} \bar{z}-2 \phi_{, z \bar{z}} \bar{\chi} \gamma_{\mu} \chi\right)+\frac{i}{2} \widehat{\bar{l}}\left(\phi_{, z} \chi-\phi_{, \bar{z}} \chi^{c}\right), \\
& D=B \phi_{, z \bar{z}}\left(|h|^{2}-\widehat{D}_{\mu} \bar{z} \widehat{D}^{\mu} z-\bar{\chi} \overleftrightarrow{D_{D}} \chi\right)-B \phi_{, z z \bar{z}}\left(\bar{h} \overline{\chi^{c}} \chi+\bar{\chi} \gamma^{\mu} \chi \widehat{D}_{\mu} z\right) \\
& -B \phi_{, z \overline{z z}}\left(h \bar{\chi} \chi^{c}+\overline{\chi^{c}} \gamma^{\mu} \chi^{c} \widehat{D}_{\mu} \bar{z}\right)+B \phi_{, z z \overline{z z}} \overline{\chi^{c}} \chi \cdot \bar{\chi} \chi^{c} \\
& -\frac{1}{2} \widehat{D}^{\mu} B \widehat{D}_{\mu} \phi-\frac{i}{2} \widehat{D}^{\mu} A\left(\phi_{, z} \widehat{D}_{\mu} z-\phi_{, \bar{z}} \widehat{D}_{\mu} \bar{z}-2 \phi_{, z} \bar{z} \bar{\chi} \gamma_{\mu} \chi\right) \\
& -\frac{1}{2}\left(\bar{l}\left(h \phi_{, z}-\phi_{, z z} \overline{\chi^{c}} \chi\right)+l\left(\bar{h} \phi_{, \bar{z}}-\phi_{, \overline{z z}} \bar{\chi} \chi^{c}\right)\right) \\
& +i \bar{\psi} \phi_{, z \bar{z}}\left(\bar{h} \chi-h \chi^{c}+\widehat{\not D} \bar{z} \chi-\widehat{D} z \chi^{c}\right) \\
& +i\left(\phi_{, z z \bar{z}} \overline{\chi^{c}} \chi \bar{\psi} \chi^{c}-\phi_{, z \overline{z z}} \bar{\chi} \chi^{c} \bar{\psi} \chi\right)-i\left(\phi_{, z \overline{z z}} \bar{\chi} \chi^{c} \bar{\chi}^{c} \psi-\phi_{, z z \bar{z}} \overline{\chi^{c}} \chi \bar{\chi} \psi\right) \\
& +\frac{i}{2}\left(\left(\phi, \bar{z} \bar{\chi}-\phi_{, z} \bar{\chi}^{c}\right) \widehat{D D} \psi-\bar{\psi} \widehat{\not D}\left(\phi_{, z} \chi-\phi_{, z} \chi^{c}\right)\right) \text {. }
\end{aligned}
$$

We can also add to the action a potential in the form of a holomorphic function $g(\Sigma)$ of the chiral multiplet $\Sigma[8$, [13]

$$
g(\Sigma)=\left(g(z), g_{, z}(z) \chi, g_{, z}(z) h-g_{,, z z}(z) \bar{\chi} \chi\right) .
$$

Coupling of a chiral multiplet $\Sigma$ can be made locally supersymmetric by extracting the $F$ term in the form according to the formula

$$
e^{-1} L_{F}=\operatorname{Re}\left(h+u z+\bar{\psi}_{\mu} \gamma^{\mu} \chi+\bar{\psi}_{\mu} \sigma^{\mu \nu} \psi_{\nu R} z\right)
$$


Applying this to the potential function $g(\Sigma)$ gives [8], [13]

$$
e^{-1} L_{F}=\frac{1}{2}\left(g_{, z} h-g_{,, z z} \bar{\chi} \chi+u g+g_{, z} \bar{\psi}_{\mu} \gamma^{\mu} \chi+g \bar{\psi}_{\mu} \sigma^{\mu \nu} \psi_{\nu R}+h . c\right) .
$$

The total action is then that of supergravity coupled to a constrained chiral multiplet $\Sigma$ with potential $g(\Sigma)$ through a Lagrange multiplier chiral multiplet $S$ with an action

$$
e^{-1} L=e^{-1} L_{S G}+e^{-1} L_{D}(S . \phi(\Sigma, \bar{\Sigma}))+e^{-1} L_{F}(g(\Sigma)) .
$$

The full Lagrangian in component form is given in the appendix equation (63).

At this point, in order to compare with mimetic gravity, we will analyze the bosonic part of the action. After integrating by parts the $\partial_{\mu} B$ term, we get

$$
\begin{aligned}
e^{-1} L^{\text {bosonic }} & =B \phi_{, z \bar{z}}|h|^{2}+\frac{1}{2} B g^{\mu \nu}\left(\phi_{, z z} \partial_{\mu} z \partial_{\nu} z+\phi_{, \bar{z}} \partial_{\mu} \bar{z} \partial_{\nu} \bar{z}\right)-\frac{i}{2} \partial^{\mu} A\left(\phi_{, z} \partial_{\mu} z-\phi_{, \bar{z}} \partial_{\mu} \bar{z}\right) \\
& -\frac{1}{2}\left(\bar{l} h \phi_{, z}+l \bar{h} \phi_{, \bar{z}}\right)+\frac{1}{3} A^{\mu}\left(i B\left(\phi_{, z} \partial_{\mu} z-\phi_{, \bar{z}} \partial_{\mu} \bar{z}\right)+\phi \partial_{\mu} A\right) \\
& +\left(\frac{1}{3} B \phi-1\right)\left(\frac{1}{2} R+\frac{1}{3}|u|^{2}-\frac{1}{3} A_{\mu} A^{\mu}\right) \\
& -\frac{1}{3}\left(\bar{u}\left(-B h \phi_{, z}+\frac{1}{2} \phi l\right)+u\left(-B \bar{h} \phi_{, \bar{z}}+\frac{1}{2} \phi \bar{l}\right)\right) \\
& +\frac{1}{2}\left(g_{, z} h+u g+\bar{g}_{, \bar{z}} \bar{h}+\overline{u g}\right) .
\end{aligned}
$$

Integrating by parts and solving the auxiliary field equations for $A, B, l, h$, and $u$ we get a coupled system of equations. We start first with the equations of the Lagrange multiplier multiplet $S$. The $B$ equation gives

$$
\begin{aligned}
0 & =\phi_{, z \bar{z}}|h|^{2}+\frac{1}{2} g^{\mu \nu}\left(\phi_{, z z} \partial_{\mu} z \partial_{\nu} z+\phi_{, \overline{z z}} \partial_{\mu} \bar{z} \partial_{\nu} \bar{z}\right)+\frac{i}{3} A^{\mu}\left(\phi_{, z} \partial_{\mu} z-\phi_{, \bar{z}} \partial_{\mu} \bar{z}\right) \\
& +\frac{1}{3} \phi\left(\frac{1}{2} R+\frac{1}{3}|u|^{2}-\frac{1}{3} A_{\mu} A^{\mu}\right)+\frac{1}{3}\left(\bar{u} h \phi_{, z}+u \bar{h} \phi_{, \bar{z}}\right) .
\end{aligned}
$$

The $A$ equation is

$$
0=\frac{i}{2} \partial^{\mu}\left(\phi_{, z} \partial_{\mu} z-\phi_{, \bar{z}} \partial_{\mu} \bar{z}\right)-\frac{1}{3} \partial^{\mu}\left(\phi A_{\mu}\right),
$$

while the $\bar{l}$ equation gives

$$
0=h \phi_{, z}+\frac{1}{3} u \phi .
$$


The equation for the auxiliary field $\bar{h}$ of the mimetic multiplet $\Sigma^{\dagger}$ gives

$$
0=B h \phi_{, z \bar{z}}-\frac{1}{2} l \phi_{, \bar{z}}+\frac{1}{3} u B \phi_{, \bar{z}}+\frac{1}{2} \bar{g}_{\bar{z}}
$$

Finally, the equations for the supergravity auxiliary fields $\bar{u}$ and $A^{\mu}$ are

$$
\begin{aligned}
& 0=\frac{1}{3}\left(\frac{B \phi}{3}-1\right) u-\frac{1}{6} \phi l+\frac{1}{3} B h \phi_{, z}+\frac{1}{2} \bar{g} \\
& 0=\left(i B\left(\phi_{, z} \partial_{\mu} z-\phi_{, \bar{z}} \partial_{\mu} \bar{z}\right)+\phi \partial_{\mu} A\right)-2\left(\frac{B \phi}{3}-1\right) A_{\mu} .
\end{aligned}
$$

We now solve this system of equations in terms of the complex mimetic field $z$. The auxiliary fields $u, A_{\mu}, h, l$ are then given by

$$
\begin{aligned}
l & =3 \frac{\bar{g}}{\phi}+6 h \frac{\phi_{, z}}{\phi^{2}}, \quad u=-3 h \frac{\phi_{, z}}{\phi}, \\
h & =\frac{\frac{3 \bar{g}}{\phi}-\frac{\bar{g}_{, \bar{z}}}{\phi_{, z}}}{2\left(B\left(\frac{\phi_{, z \bar{z}}}{\phi, \bar{z}}-\frac{\phi_{, z}}{\phi}\right)-3 \frac{\phi_{, z}}{\phi^{2}}\right)}, \\
A_{\mu} & =\frac{1}{2\left(\frac{B \phi}{3}-1\right)}\left(i B\left(\phi_{, z} \partial_{\mu} z-\phi, \bar{z} \partial_{\mu} \bar{z}\right)+\phi \partial_{\mu} A\right) .
\end{aligned}
$$

Substituting these values into equations (33) and (34) as well as using the $z$ equations of motion, we get differential equations sufficient to determine the fields $A, B$ and $z$. However, unlike gravity with mimetic field, the auxiliary fields in supergravity, $u$ and $A_{\mu}$ do contribute to the effective action and would cause both the phase factor of the field $z$ as well as the field $A$ to propagate implying that the bosonic sector will have two degrees of freedom for the graviton and two degrees of freedom for the scalar fields. The solutions depend on the choice of functions $\phi(z, \bar{z})$ and $g(z)$ and for reasonable choices of these functions the equations will simplify. In particular we can use a linear potential

$$
g(z)=m^{2}(z+\beta),
$$

the same form used for the hidden sector breaking local supersymmetry spontaneously [8], [11. Similarly we can use a simple choice for the function $\phi(z, \bar{z})$ such as

$$
\phi(z, \bar{z})=\phi_{0} \exp (-c \bar{z} z),
$$

which would greatly simplify the equations. We notice that the $B$ equation (33) gives a constraint on the combination $g^{\mu \nu}\left(\phi_{, z z} \partial_{\mu} z \partial_{\nu} z+\phi_{, z z} \partial_{\mu} \bar{z} \partial_{\nu} \bar{z}\right)$, 
instead of $g^{\mu \nu} \phi_{, z \bar{z}} \partial_{\mu} z \partial_{\nu} \bar{z}$. This should not be necessarily a problem because the metric $g^{\mu \nu}$ has a Lorentzian signature and derivatives with respect to time and space coordinates come with opposite signs. Thus constraints of this type could always be satisfied by allowing the real and imaginary parts to depend on both time and space coordinates. This is a situation encountered in our construction of inhomogeneous dark energy [15]. In the fermionic sector the Lagrange multiplier fermion $\psi$ constrains the mimetic field $\chi$ which does couple to the gravitino $\psi_{\mu}$. A careful Hamiltonian analysis along the lines of [16] is needed to show explicitly which combination of fields propagate and which ones correspond to mimetic dark matter in the form of two scalars. The structure of the action indicates that one can use the potential function $g(z)$ to break supersymmetry. In this case the singlet field $\Sigma$ does act as the hidden sector in supergravity triggering the breakdown of supersymmetry [8, [11]. In the absence of supersymmetry breaking the fermionic degrees of freedom match the bosonic ones and one can see from the action of the constrained fermionic system $(\psi, \chi)$ that a massless chiral fermion propagate. When supersymmetry is broken spontaneously the massless fermion will be absorbed by the gravitino which becomes massive with four degrees of freedom matching the four degrees of the graviton plus two scalars. We will further elaborate on this in section 4 when we consider spontaneous breaking of supersymmetry.

\section{Real linear multiplet as Lagrange multiplier}

A real linear multiplet is real vector superfield satisfying the constraint

$$
D^{2} L=0=\bar{D}^{2} L
$$

This implies that the surviving components of the vector multiplet take the form [17]

$$
L=\left(A, \psi, b_{\mu}, 0,-\widehat{D} \psi,-\square^{P} A\right),
$$

where $b_{\mu}$ is a divergence free vector $\partial^{\mu} b_{\mu}=0$. We now couple the linear multiplet $L$ to the symmetric product function $\phi\left(\Sigma, \Sigma^{\dagger}\right)$ to obtain a vector 
multiplet $V$ with components

$$
\begin{aligned}
& C=\frac{1}{2} A \phi, \quad \zeta=i A\left(\phi_{, z} \chi-\phi_{, \bar{z}} \chi^{c}\right)+\frac{1}{2} \phi \psi, \\
& v=-A\left(\phi_{, z} h-\phi_{, z z} \overline{\chi^{c}} \chi\right)-\frac{i}{2} \bar{\psi}_{R}\left(\phi_{, z} \chi_{L}-\phi_{, \bar{z}} \chi_{L}^{c}\right)+\frac{i}{2}\left(\phi_{, \bar{z}} \bar{\chi}_{R}-\phi_{, z} \bar{\chi}_{R}\right) \psi_{L}, \\
& V_{\mu}=\frac{i}{2} A\left(\phi_{, z} \widehat{D}_{\mu} z-\phi_{, \bar{z}} \widehat{D}_{\mu} \bar{z}-2 \phi_{, z z} \bar{\chi} \gamma_{\mu} \chi\right)+\frac{1}{2} \phi b_{\mu}+\frac{1}{2} \bar{\psi} \gamma_{\mu} \gamma_{5}\left(\phi_{, z} \chi-\phi_{, \bar{z}} \chi^{c}\right), \\
& \lambda=A \phi_{, z \bar{z}}\left(-i \bar{h} \chi+i h \chi^{c}-i \widehat{\not D} \bar{z} \chi+i \widehat{\not D} z \chi^{c}\right)-\frac{1}{2} \phi \widehat{\not D} \psi \\
& -\frac{i}{2} \widehat{\not D} A\left(\phi_{, z} \chi-\phi_{, \bar{z}} \chi^{c}\right)-\frac{1}{4} \widehat{\not D} \phi \psi-i A\left(\phi_{, z z \bar{z}} \overline{\chi^{c}} \chi \chi^{c}-\phi_{, z \overline{z z}} \bar{\chi} \chi^{c} \chi\right) \\
& -\frac{1}{2} \gamma_{5} \gamma^{\mu}\left(b_{\mu}\left(\phi_{, z} \chi-\phi_{, \bar{z}} \chi^{c}\right)+\frac{1}{2}\left(\phi_{, z} \widehat{D}_{\mu} z-\phi_{, \bar{z}} \widehat{D}_{\mu} \bar{z}-2 \phi_{, z \bar{z}} \bar{\chi} \gamma_{\mu} \chi\right) \psi\right) \\
& -\frac{1}{2}\left(\phi_{, \bar{z}} \bar{h}-\phi, \overline{z z} \bar{\chi} \chi^{c}\right) \psi \\
& D=A \phi_{, z \bar{z}}\left(|h|^{2}-\widehat{D}_{\mu} \bar{z} \widehat{D}^{\mu} z-\bar{\chi} \overleftrightarrow{\grave{D}} \chi\right)-A \phi_{, z z \bar{z}}\left(h^{*} \overline{\chi^{c}} \chi+\bar{\chi} \gamma^{\mu} \chi \widehat{D}_{\mu} z\right) \\
& -A \phi_{, z \overline{z z}}\left(h \bar{\chi} \chi^{c}+\overline{\chi^{c}} \gamma^{\mu} \chi^{c} \widehat{D}_{\mu} \bar{z}\right)+A \phi_{, z z \overline{z z}} \overline{\chi^{c}} \chi \cdot \bar{\chi} \chi^{c}-\frac{1}{2} \phi \square^{P} A-\frac{1}{2} \widehat{D}^{\mu} A \widehat{D}_{\mu} \phi \\
& -\frac{i}{2} b^{\mu}\left(\phi_{, z} \widehat{D}_{\mu} z-\phi_{, z} \widehat{D}_{\mu} \bar{z}-2 \phi_{, z} \bar{z} \gamma_{5} \gamma_{\mu} \chi\right)+i \phi_{, z \bar{z}} \bar{\psi}\left(\bar{h} \chi-h \chi^{c}+\widehat{D} \bar{z} \chi-\widehat{D} z \chi^{c}\right) \\
& -i \phi_{, z \bar{z}}\left(h \bar{\chi}-\bar{h} \overline{\chi^{c}}+\widehat{D D} z \bar{\chi}-\widehat{D} \bar{z} \overline{\chi^{c}}\right) \psi+\frac{i}{2}\left(\phi_{, \bar{z}} \bar{\chi}-\phi_{, z} \overline{\chi^{c}}\right) \widehat{\not D} \psi \\
& -\frac{i}{2} \bar{\psi} \widehat{\not D}\left(\phi_{, z} \chi-\phi_{, \bar{z}} \chi^{c}\right)+i\left(\phi_{, z z \bar{z}}\left(\overline{\chi^{c}} \chi\right) \bar{\psi} \chi^{c}-\phi_{, z \overline{z z}}\left(\bar{\chi} \chi^{c}\right) \bar{\psi} \chi\right) \\
& -i\left(\phi_{, z \overline{z z}} \bar{\chi} \chi^{c} \bar{\chi}^{c} \psi-\phi_{, z z \bar{z}} \overline{\chi^{c}} \chi \bar{\chi} \psi\right) \text {. }
\end{aligned}
$$

This multiplet is coupled to supergravity using the formula (21). In addition we add the potential term $g(\Sigma)$ using formula (31). The full Lagrangian in this case is given by

$$
e^{-1} L=e^{-1} L_{S G}+e^{-1} L_{D}(L . \phi(\Sigma, \bar{\Sigma}))+e^{-1} L_{F}(g(\Sigma)) .
$$


The component form of this action is given in the appendix equation (64). Collecting all bosonic terms we obtain the Lagrangian

$$
\begin{aligned}
e^{-1} L^{\text {bosonic }} & =A \phi_{, z \bar{z}}\left(|h|^{2}-\partial_{\mu} \bar{z} \partial^{\mu} z\right)-\frac{i}{2} b^{\mu}\left(\phi_{, z} \partial_{\mu} z-\phi_{, \bar{z}} \partial_{\mu} \bar{z}\right) \\
& +\frac{A}{3}\left(\bar{u} \phi_{, z} h+u \phi_{, \bar{z}} \bar{h}\right)+\frac{1}{3} A^{\mu}\left(i A\left(\phi_{, z} \partial_{\mu} z-\phi_{, \bar{z}} \partial_{\mu} \bar{z}\right)+\phi b_{\mu}\right) \\
& +\left(\frac{1}{3} A \phi-1\right)\left(\frac{1}{2} R+\frac{1}{3}|u|^{2}-\frac{1}{3} A_{\mu} A^{\mu}\right)+\frac{1}{2}\left(g_{, z} h+u g+\bar{g}_{, \bar{z}} \bar{h}+\overline{u g}\right)
\end{aligned}
$$

where we have integrated by parts the $\widehat{D}^{\mu} A$ term to cancel the $\square^{P} A$ term leaving only the $A \widehat{D}_{\mu} \bar{z} \widehat{D}^{\mu} z$ kinetic term just as in gravity with mimetic matter. We first vary the Lagrange multiplier $A$ to obtain

$$
\begin{aligned}
0 & =\phi_{, z \bar{z}}\left(|h|^{2}-\partial_{\mu} \bar{z} \partial^{\mu} z\right)+\frac{1}{3}\left(\bar{u} \phi_{, z} h+u \phi_{, \bar{z}} \bar{h}\right)+\frac{i}{3} A^{\mu}\left(\phi_{, z} \partial_{\mu} z-\phi_{, z} \partial_{\mu} \bar{z}\right) \\
& +\frac{1}{3} \phi\left(\frac{1}{2} R+\frac{1}{3}|u|^{2}-\frac{1}{3} A_{\mu} A^{\mu}\right)
\end{aligned}
$$

Next we vary the divergence free vector $b_{\mu}$ to obtain

$$
0=\frac{i}{2}\left(\phi_{, z} \partial_{\mu} z-\phi_{, \bar{z}} \partial_{\mu} \bar{z}\right)-\frac{1}{3}\left(\phi A_{\mu}\right)+\partial_{\mu} \alpha
$$

where $\alpha$ is determined from transversality of $b_{\mu}$. We next vary the auxiliary field $\bar{h}$ of the chiral multiplet $\Sigma^{\dagger}$ to get

$$
0=A\left(\phi_{, z \bar{z}} h+\frac{1}{3} u \phi_{, \bar{z}}\right)+\frac{1}{2} \bar{g}_{, \bar{z}}
$$

Finally we vary the supergravity auxiliary fields $\bar{u}$ and $A^{\mu}$ to obtain, respectively

$$
\begin{aligned}
& 0=\frac{1}{3}\left(\frac{1}{3} A \phi-1\right) u+\frac{A}{3} \phi_{, z} h+\frac{1}{2} \bar{g} \\
& 0=\left(i A\left(\phi_{, z} \partial_{\mu} z-\phi_{, \bar{z}} \partial_{\mu} \bar{z}\right)+\phi b_{\mu}\right)-2\left(\frac{1}{3} A \phi-1\right) A_{\mu}
\end{aligned}
$$

These equations allow us to solve for $u, A_{\mu}, h$ and $b_{\mu}$ in terms of $A$ and $z$ which are constrained by equations (53) and the $z$ equation. These are 
given by

$$
\begin{aligned}
h & =-\frac{1}{2\left(\frac{A}{3}\left(\phi \phi_{, z \bar{z}}-\phi_{, z} \phi_{, \bar{z}}\right)-\phi_{, z \bar{z}}\right)}\left(\left(\frac{\phi}{3}-\frac{1}{A}\right) g_{, \bar{z}}^{*}-\phi_{, \bar{z}} g^{*}\right) \\
u & =\frac{1}{2\left(\frac{A}{3}\left(\phi \phi_{, z \bar{z}}-\phi_{, z} \phi_{, \bar{z}}\right)-\phi_{, z \bar{z}}\right)}\left(\phi_{, z} g_{, \bar{z}}^{*}-3 \phi_{, z \bar{z}} g^{*}\right) \\
A_{\mu} & =\frac{3}{\phi} \partial_{\mu} \alpha+\frac{3 i}{2 \phi}\left(\phi_{, z} \partial_{\mu} z-\phi_{, \bar{z}} \partial_{\mu} \bar{z}\right) \\
b_{\mu} & =\left(\frac{A}{\phi}-\frac{3}{\phi^{2}}\right) \partial_{\mu} \alpha-\frac{3 i}{\phi}\left(\phi_{, z} \partial_{\mu} z-\phi_{, \bar{z}} \partial_{\mu} \bar{z}\right)
\end{aligned}
$$

Noting that the Lagrange multiplier multiplet couples linearly, all terms in such couplings drop out from the action leaving only the supergravity action

$$
-\left(\frac{e}{2} R+\frac{e}{3}|u|^{2}-\frac{e}{3} A_{\mu} A^{\mu}+\frac{1}{2} \bar{\psi}_{\mu} R^{\mu}\right)
$$

where $u$ and $A_{\mu}$ are given in equations (59) and (60). The field $A$ will be determined from the components of the Einstein tensor $G_{\mu \nu}$. Again we obtain four dynamical degrees of freedom, two for the graviton and two for the scalar fields $\alpha$ and imaginary part of $z$.

\section{Conclusions and road map to future work}

Gravity with mimetic dark matter was shown to be a versatile model solving many outstanding problems. To name few of the advantages we mention first the generation of mimetic dark matter in the form of dust or geometric extrinstic curvature contributions to the energy-momentum tensor [1], 4]. Including appropriate functions $f(\square \phi)$ in the action lead to non-singular cosmological and black hole solutions. Also, with the aid of the mimetic field $\phi$ it is possible to construct Horava type models of renormalizable gravity without breaking diffeomorphism invariance [18]. All these gains were achieved without introducing new degrees of freedom to the graviton, except for half a degree corresponding to the longitudinal mode. On the other hand supersymmetry have attracted a lot of attention and is looked at as the most viable theory beyond the Standard Model. In particular $N=1$ locally supersymmetric theories can result as the low energy limit of consistent superstring models. As supersymmetry is not observed in nature it must be broken spontaneously. This is usually done through a hidden sector represented by a singlet chiral supermultiplet [8], [11]. It is then natural to combine both ideas and ask the question whether it is possible to 
supersymmetrize the action of gravity with mimetic dark matter. Although the gravity action was first constructed by maintaining the symmetry (1), it proved easier to equivalently impose the constraint equation(3) [2]. In this work we imposed a constraint on the symmetric product $\Sigma^{\dagger} \Sigma$ of a chiral supermultiplet $\Sigma=(z, \chi, h)$ with its conjugate $\Sigma^{\dagger}$ first using a chiral supermultiplet $S=(A+i B, \psi, l)$ as a Lagrange multiplet and later using instead a linear multiplet $L=\left(C, \psi, b_{\mu}\right)$. We have constructed the most general coupling of this system to supergravity represented by the multiplet $S G=\left(e_{\mu}^{a}, \psi_{\mu}, u, A_{\mu}\right)$.

We have shown that the mimetic dark matter supermultiplet do couple to the auxiliary fields of supergravity. These will then contribute to the action of auxiliary fields and in general will have non-trivial solutions. In addition, the gravitino couples to the superpotential and for particular solutions for the field $z$ satisfying the constraint equations, preliminary work strongly indicates that supersymmetry will be broken spontaneously. The aim of future work is to study that the system $S, \Sigma, S G$ or $L, \Sigma, S G$ in presence of a superpotential $g(z)$, and to determine the spectrum. In particular a careful analysis is needed to check how the gravitino field $\psi_{\mu}$ absorbs the Goldstino field. It is known that in generic models of local supersymmetry a singlet field with appropriate superpotential function $g(z)$ can break supersymmetry spontaneously and that the gravitino field absorbs the Goldistino to become massive [8], [11, [13]. The main difference here is that supergravity is considered as part of a constrained system. This system consists of the supergravity multiplet $\left(e_{\mu}^{a}, \psi_{\mu}, A_{\mu}, u\right)$ of 16 bosonic and 16 fermionic component fields and a chiral multiplet $(z, \chi, h)$ of 4 bosonic and 4 fermionic component fields as well as the Lagrange multiplier chiral multiplet $(A+i B, \psi, l)$ or linear multiplet $\left(A, \psi, b_{\mu}\right)$. This system will effectively have the same number of independent degrees of freedom as that of a spontaneously broken supergravity multiplet with four fermionic degrees for the massive gravitino, and four bosonic degrees, two for the graviton and two for the scalar fields. However, only the model with Linear multiplet yields a constraint on the kinetic term of the form $g^{\mu \nu} \phi_{, z \bar{z}} \partial_{\mu} z \partial_{\nu} \bar{z}$ while the model with multiplet $S$ gives a constraint on the combination $g^{\mu \nu}\left(\phi_{, z z} \partial_{\mu} z \partial_{\nu} z+\phi_{, \bar{z}} \partial_{\mu} \bar{z} \partial_{\nu} \bar{z}\right)$. Thus the model with coupling to the Linear multiplet is more in line with the non-supersymmetric case. The supersymmetric case is, however, much more complicated because of the presence of the auxiliary fields $u, A_{\mu}, h$, as these couple non-trivially to the system $(S, \Sigma)$ or $(L, \Sigma)$. Imposing and solving the constraints coming from the Lagrange multiplier multiplet and eliminating the auxiliary fields give rise to an action containing only the physical degrees of freedom. For general 
functions $\phi(z, \bar{z})$ and $g(z)$ the resulting action is complicated. It should, however, be possible to simplify the form by performing some redefinitions along the lines of the results in [8], [12, [13]. What is expected to be gained here is that the combination of a chiral multiplet and a Lagrange multiplier multiplet could act as the hidden sector responsible for supersymmetry breaking and for the appearance of mimetic dark matter in the form of two scalar fields. These steps will be undertaken in future work where the aim will be to present a realistic model where the Goldistino field will be the supersymmetric partner of mimetic dark matter with details on the local supersymmetry breaking and the actual field combinations representing the dynamical degrees of freedom as well as the fields determining dark matter.

\section{Appendix}

In this appendix we give the full Lagrangian for supergravity with mimetic dark matter chiral multiplet $\Sigma=(z, \chi, h)$ that can also act as the hidden sector for spontaneous supersymmetry breaking. In the first case the Lagrange multiplier multiplet is a left handed chiral multiplet $S=(A+i B, \psi, l)$ embedded in a real vector multiplet. The full Lagrangian (32) is given in 
component form by

$$
\begin{aligned}
& e^{-1} L=\left(\frac{1}{3} B \phi-1\right)\left(\frac{1}{2} R+\frac{1}{3}|u|^{2}-\frac{1}{3} A_{\mu} A^{\mu}+\frac{1}{2} e^{-1} \bar{\psi}_{\mu} R^{\mu}\right) \\
& +B \phi_{, z \bar{z}}\left(|h|^{2}-\widehat{D}_{\mu} \bar{z} \widehat{D}^{\mu} z-\bar{\chi} \overleftrightarrow{\mathbb{D}} \chi\right)-B \phi_{, z z \bar{z}}\left(h^{*} \overline{\chi^{c}} \chi+\bar{\chi} \gamma^{\mu} \chi \widehat{D}_{\mu} z\right) \\
& -B \phi_{, z \overline{z z}}\left(h \bar{\chi} \chi^{c}+\overline{\chi^{c}} \gamma^{\mu} \chi^{c} \widehat{D}_{\mu} \bar{z}\right)+B \phi_{, z z \overline{z z}} \overline{\chi^{c}} \chi \cdot \bar{\chi} \chi^{c} \\
& -\frac{1}{2} \widehat{D}^{\mu} B \widehat{D}_{\mu} \phi-\frac{i}{2} \widehat{D}^{\mu} A\left(\phi_{, z} \widehat{D}_{\mu} z-\phi_{, \bar{z}} \widehat{D}_{\mu} \bar{z}-2 \phi_{, z \bar{z}} \bar{\chi} \gamma_{\mu} \chi\right) \\
& -\frac{1}{2}\left(\bar{l}\left(h \phi_{, z}-\phi_{, z z} \overline{\chi^{c}} \chi\right)+l\left(\bar{h} \phi_{, \bar{z}}-\phi_{, \bar{z}} \bar{\chi} \chi^{c}\right)\right)+i \bar{\psi} \phi_{, z \bar{z}}\left(h^{*} \chi-h \chi^{c}+\widehat{\not D} \bar{z} \chi-\widehat{D} z \chi^{c}\right) \\
& +i\left(\phi_{, z z \bar{z}} \overline{\chi^{c}} \chi \bar{\psi} \chi^{c}-\phi_{, z \overline{z z}} \bar{\chi} \chi^{c} \bar{\psi} \chi\right)-i\left(\phi_{, z \overline{z z}} \bar{\chi} \chi^{c} \bar{\chi}^{c} \psi-\phi_{, z z \bar{z}} \overline{\chi^{c}} \chi \bar{\chi} \psi\right) \\
& +\frac{i}{2}\left(\left(\phi_{, z} \bar{\chi}-\phi_{, z} \bar{\chi}^{c}\right) \widehat{\not D} \psi-\bar{\psi} \widehat{\not D}\left(\phi_{, z} \chi-\phi_{, \bar{z}} \chi^{c}\right)\right) \\
& -\frac{i}{2} \bar{\psi}_{\mu} \gamma_{5} \gamma^{\mu}\left[-i B \phi_{, z \bar{z}}\left(\left(h^{*} \chi-h \chi^{c}\right)+\widehat{D} \bar{z} \chi-\widehat{\not D} z \chi^{c}\right)\right. \\
& -i B\left(\phi_{, z z \bar{z}}\left(\overline{\chi^{c}} \chi\right) \chi^{c}-\phi_{, z \overline{z z}}\left(\bar{\chi} \chi^{c}\right) \chi\right)-\frac{i}{2} \widehat{\not D} B\left(\phi_{, z} \chi-\phi_{, \bar{z}} \chi^{c}\right) \\
& -\frac{1}{4} \widehat{D D} \phi \psi-\frac{1}{2} \gamma_{5} \gamma^{\mu}\left(\phi_{, z} \chi-\phi_{, \bar{z}} \chi^{c}\right) \widehat{D}_{\mu} A-\frac{1}{2}\left(\phi_{, \bar{z}} \bar{h} \widehat{-\phi_{, z z}} \overline{\chi^{c}} \chi\right) \psi \\
& \left.-\frac{1}{4} \gamma_{5} \gamma^{\mu} \psi\left(\phi_{, z} \widehat{D}_{\mu} z-\phi_{, \bar{z}} \widehat{D}_{\mu} \bar{z}-2 \phi_{, z} \bar{z} \bar{\chi} \gamma_{\mu} \chi\right)+\frac{i}{2} \widehat{\bar{l}}\left(\phi_{, z} \chi-\phi_{, z} \chi^{c}\right)\right] \\
& +\frac{2}{3}\left(A^{\mu}+\frac{3 i}{8} e^{-1} \epsilon^{\mu \rho \sigma \tau} \bar{\psi}_{\rho} \gamma_{\tau} \psi_{\sigma}\right)\left[\frac{i}{2} B\left(\phi_{, z} \widehat{D}_{\mu} z-\phi_{, \bar{z}} \widehat{D}_{\mu} \bar{z}-2 \phi_{, z \bar{z}} \bar{\chi} \gamma_{\mu} \chi\right)\right. \\
& \left.+\frac{1}{2} \phi \widehat{D}_{\mu} A+\frac{1}{2} \bar{\psi} \gamma_{\mu} \gamma_{5}\left(\phi_{, z} \chi-\phi_{, \bar{z}} \chi^{c}\right)\right] \\
& +\frac{i}{3} e^{-1}\left(-i B\left(\phi_{, \bar{z}} \bar{\chi}-\phi_{, z} \bar{\chi}^{c}\right)+\frac{1}{2} \phi \bar{\psi}\right) \gamma_{5} \gamma_{\mu} R^{\mu} \\
& +\frac{i}{8} e^{-1} \epsilon^{\mu \nu \rho \sigma} \bar{\psi}_{\mu} \gamma_{\nu} \psi_{\rho}\left(-i B\left(\phi, \bar{z} \bar{\chi}-\phi_{, z} \bar{\chi}^{c}\right)+\frac{1}{2} \phi \bar{\psi}\right) \psi_{\sigma} \\
& +\frac{1}{3}\left(\bar{u}\left(-B\left(h \phi_{, z}-\phi_{, z z} \overline{\chi^{c}} \chi\right)+\frac{1}{2} \phi l-\frac{i}{2} \bar{\psi}_{R}\left(\phi_{, z} \chi_{L}-\phi_{, \bar{z}} \chi_{L}^{c}\right)+\frac{i}{2}\left(\phi_{, \bar{z}} \bar{\chi}_{R}-\phi_{, z}{\overline{\chi^{c}}}_{R}\right) \psi_{L}\right)+h . c\right) \\
& +\frac{1}{2}\left(g_{, z} h-g_{,, z z} \bar{\chi} \chi+u g+g_{, z} \bar{\psi}_{\mu} \gamma^{\mu} \chi+g \bar{\psi}_{\mu} \sigma^{\mu \nu} \psi_{\nu R}+h . c\right)
\end{aligned}
$$

The second Lagrangian corresponds to the case where the Lagrange multiplier supermultiplet is a linear multiplet $L=(A, \psi, b)$ embedded in a real vector multiplet. When expressed in terms of components the full 
Lagrangian (51) becomes

$$
\begin{aligned}
& e^{-1} L=\left(\frac{1}{3} A \phi-1\right)\left(\frac{1}{2} R+\frac{1}{3}|u|^{2}-\frac{1}{3} A_{\mu} A^{\mu}+\frac{1}{2} e^{-1} \bar{\psi}_{\mu} R^{\mu}\right) \\
& +A \phi_{, z \bar{z}}\left(|h|^{2}-\widehat{D}_{\mu} \bar{z} \widehat{D}^{\mu} z-\bar{\chi} \overleftrightarrow{\widehat{D}} \chi\right)-A \phi_{, z z \bar{z}}\left(\bar{h} \overline{\chi^{c}} \chi+\bar{\chi} \gamma^{\mu} \chi \widehat{D}_{\mu} z\right) \\
& -A \phi_{, z \overline{z z}}\left(h \bar{\chi} \chi^{c}+\overline{\chi^{c}} \gamma^{\mu} \chi^{c} \widehat{D}_{\mu} \bar{z}\right)+A \phi_{, z z \overline{z z}}\left(\overline{\chi^{c}} \chi\right)\left(\bar{\chi} \chi^{c}\right)-\frac{1}{2} \phi \square^{P} A-\frac{1}{2} \widehat{D}^{\mu} A \widehat{D}_{\mu} \phi \\
& -\frac{i}{2} b^{\mu}\left(\phi_{, z} \widehat{D}_{\mu} z-\phi_{, \bar{z}} \widehat{D}_{\mu} \bar{z}-2 \phi_{, z} \bar{z} \bar{\chi} \gamma_{5} \gamma_{\mu} \chi\right)+i \phi_{, z \bar{z}} \bar{\psi}\left(\bar{h} \chi-h \chi^{c}+\widehat{D} \bar{z} \chi-\widehat{D} z \chi^{c}\right) \\
& -i \phi_{, z \bar{z}}\left(h \bar{\chi}-\bar{h} \overline{\chi^{c}}+\widehat{D D} z \bar{\chi}-\widehat{D D} \bar{z} \overline{\chi^{c}}\right) \psi+\frac{i}{2}\left(\phi, \bar{z} \bar{\chi}-\phi_{, z} \overline{\chi^{c}}\right) \widehat{\not D} \psi \\
& -\frac{i}{2} \bar{\psi}_{\mu} \gamma_{5} \gamma^{\mu}\left[A \phi_{, z \bar{z}}\left(-i \bar{h} \chi+i h \chi^{c}-i \widehat{\not D} \bar{z} \chi+i \widehat{D D} z \chi^{c}\right)-\frac{1}{2} \phi \widehat{\not D} \psi\right. \\
& -\frac{i}{2} \widehat{\not D} A\left(\phi_{, z} \chi-\phi, \bar{z} \chi^{c}\right)-\frac{1}{4} \widehat{\not D} \phi \psi-i A\left(\phi_{, z z \bar{z}} \overline{\chi^{c}} \chi \chi^{c}-\phi_{, z \overline{z z}} \bar{\chi} \chi^{c} \chi\right) \\
& \left.-\frac{1}{2} \gamma_{5} \gamma^{\mu}\left(b_{\mu}\left(\phi_{, z} \chi-\phi_{, \bar{z}} \chi^{c}\right)+\frac{1}{2}\left(\phi_{, z} \widehat{D}_{\mu} z-\phi_{, \bar{z}} \widehat{D}_{\mu} \bar{z}-2 \phi_{, z \bar{z}} \bar{\chi} \gamma_{\mu} \chi\right) \psi\right)-\frac{1}{2}\left(\phi_{, \bar{z}} \bar{h}-\phi_{, \bar{z} z} \bar{\chi} \chi^{c}\right) \psi\right] \\
& +\frac{2}{3}\left(A^{\mu}+\frac{3 i}{8} e^{-1} \epsilon^{\mu \rho \sigma \tau} \bar{\psi}_{\rho} \gamma_{\tau} \psi_{\sigma}\right)\left[\frac{i}{2} A\left(\phi_{, z} \widehat{D}_{\mu} z-\phi_{, \bar{z}} \widehat{D}_{\mu} \bar{z}-2 \phi_{, z \bar{z}} \bar{\chi} \gamma_{\mu} \chi\right)\right. \\
& \left.+\frac{1}{2} \phi b_{\mu}+\frac{1}{2} \bar{\psi} \gamma_{\mu} \gamma_{5}\left(\phi_{, z} \chi-\phi, \bar{z} \chi^{c}\right)\right] \\
& +\frac{i}{3} e^{-1}\left(-i A\left(\phi, \bar{z} \bar{\chi}-\phi_{, \bar{z}} \bar{\chi}^{c}\right)+\frac{1}{2} \phi \bar{\psi}\right) \gamma_{5} \gamma_{\mu} R^{\mu} \\
& +\frac{i}{8} e^{-1} \epsilon^{\mu \nu \rho \sigma} \bar{\psi}_{\mu} \gamma_{\nu} \psi_{\rho}\left(-i A\left(\phi_{, \bar{z}} \bar{\chi}-\phi_{, \bar{z}} \bar{\chi}^{c}\right)+\frac{1}{2} \phi \bar{\psi}\right) \psi_{\sigma} \\
& -\frac{1}{3}\left(\bar{u}\left(-A\left(\phi_{, z} h-\phi_{, z z} \overline{\chi^{c}} \chi\right)-\frac{i}{2} \bar{\psi}_{R}\left(\phi_{, z} \chi_{L}-\phi_{, \bar{z}} \chi_{L}^{c}\right)+\frac{i}{2}\left(\phi_{, \bar{z}} \bar{\chi}_{R}-\phi_{, z}{\overline{\chi^{c}}}_{R}\right) \psi_{L}\right)+h . c\right) \\
& +\frac{1}{2}\left(g_{, z} h-g_{,, z z} \bar{\chi} \chi+u g+g_{, z} \bar{\psi}_{\mu} \gamma^{\mu} \chi+g \bar{\psi}_{\mu} \sigma^{\mu \nu} \psi_{\nu R}+h . c\right)
\end{aligned}
$$

Acknowledgement 1 This research is supported in parts by the National Science Foundation Grant No. Phys-1912998.

\section{References}

[1] A. H. Chamseddine and V. Mukhanov, Mimetic Dark Matter, JHEP 1311 (2013) 135. 
[2] A. Golovnev, On the Recently Proposed Mimetic Dark Matter, Phys. Lett. B728 (2014) 39.

[3] A. H. Chamseddine, V. Mukhanov and A. Vikman, Cosmology with Mimetic Matter, JCAP 1406 (2014) 017.

[4] A. H. Chamseddine and V. Mukhanov, Resolving Cosmological Singularities, JCAP 1703

[5] A. H. Chamseddine and V. Mukhanov, Nonsingular Black Hole, Eur. Phys. J. C77 (2017) 83.

[6] A. H. Chamseddine, V. Mukhanov and T. Russ, Asymptotically Free Mimetic Gravity, Eur. Phys. J. C79 (2019) 558.

[7] A. H. Chamseddine, V. Mukhanov and T. Russ, Black hole remnants, JHEP (2019) 104.

[8] E. Cremmer, B. Julia, J. Scherk, S. Ferrara, L. Girardello and P. van Niewenhuizen, Spontaneous symmetry breaking and Higgs effect in supergravity without cosmological constant, Nucl. Phys. B147 (1979) 105.

[9] K. Stelle and P. C. West, Relation between vector and scalar multiplets and gauge invariance in supergravity, Nucl. Phys. B145 (1978) 175.

[10] E. Cremmer, S. Ferrara, L. Girardello and A. van Proeyen, Coupling supersymmetric Yang-Mills theories to supergravity, Phys. Lett. 116B, (1982) 231.

[11] A. H. Chamseddine, R. Arnowitt and P. Nath, Locally supersymmetric grand unification, Phys. Rev. Lett. 49 (1982) 970.

[12] E. Cremmer, S. Ferrara, L. Girardello and A. van Proeyen, YangMills theories with local supersymmetry: Lagrangian, transformation laws and super-Higgs effect, Nucl. Phys. B212 (1983) 413. (2017) 009.

[13] P. Nath, R. Arnowitt and A. H. Chamseddine, Applied N=1 Supergravity, World Scientific, 1984.

[14] P. van Niewenhuizen, Lectures in supergravity theory, in Recent developments in gravitation, Cargese 1978, editors M. Levy and S. Deser, Plenum Press 1979.

[15] A. H. Chamseddine and V. Mukhanov, Inhomogeneous dark energy, JCAP 02 (2016) 040. 
[16] O. Malaeb, Hamiltonian formulation of mimetic gravity, Phys. Rev. D19 (2015) 10.

[17] C. Aulakh, J. -P. Derendinger and S. Ouvry, On the reducibility of $16+16$ supergravity, Phys. Lett. B169 (1986) 201.

[18] A. H. Chamseddine, V. Mukhanov and T. Russ, Mimetic Horava gravity, Phys. Lett. B798 (2019) 134939. 